\title{
Stealth dicing of sapphire wafers with near infra-red femtosecond pulses
}

\author{
Amit Yadav $^{1} \cdot$ Hani Kbashi $^{1} \cdot$ Stanislav Kolpakov $^{1} \cdot$ Neil Gordon $^{1} \cdot$ Kaiming Zhou $^{1}$. \\ Edik U. Rafailov ${ }^{1}$
}

Received: 5 December 2016 / Accepted: 23 March 2017 / Published online: 25 April 2017

(c) The Author(s) 2017. This article is an open access publication

\begin{abstract}
The quality of the reflecting faces after dicing is critical for the fabrication of efficient and stable laser diodes emitting in the green-violet region. However, highquality faces can be difficult to achieve for devices grown on a sapphire substrate as this material is difficult to cleave cleanly. We have therefore investigated a technology known as "stealth dicing". The technology uses a pulsed laser to damage a plane of material inside of the wafer due to multiphoton absorption instead of cutting through the wafer surface. If the damage is induced in a line of stress points, the sample can then be cleaved easily along the damaged plane to leave a high-quality surface. The use of this technique also reduces thermal damage and debris.
\end{abstract}

\section{Introduction}

Semiconductor-based diodes covering the visible region of the emission spectrum are in increasing demand for a variety of applications. Light-emitting diodes (LEDs) in the wavelength range $300-550 \mathrm{~nm}$ are realized with $\mathrm{InGaN} /$ $\mathrm{GaN}$ and are grown predominantly on sapphire substrates. After a LED structure is printed on the surface of the sapphire wafer, the wafer needs to be diced to separate the micro-devices for encapsulation. The quality of the reflecting faces after dicing is critical for the fabrication of efficient and stable LEDs emitting in the green-violet region (GVR) [1, 2]. However, sapphire is fragile and hard (the

Amit Yadav

yadava@aston.ac.uk

1 School of Engineering and Applied Science, Aston University, Aston Triangle, Birmingham B4 7ET, UK
Mohs hardness index of 9) [3], and these properties make sapphire dicing a challenging affair.

The mechanical separation of micro-devices, sometimes called "chips", using a diamond saw (diamond scribing) was the first technology used for chip separation and this technique was also a common choice to perform dicing of sapphire substrates. Since scribing is a contact process, there are critical issues of debris, delamination, chipping and heating of the wafer because of friction. Furthermore, diamond scribing is slow, produces a wide kerf with a width typically between $\sim 100$ and $200 \mu \mathrm{m}$ and has a low yield [4]. What is more, the quality of the surface (see Fig. 1a) after sawing is not acceptable even for light emitting diodes (LEDs).

The laser-based technologies for dicing are based on light-matter interactions and can be grouped into two types: laser scribing and the stealth dicing. These technologies resolve many of the drawbacks of diamond scribing and today they are the prevalent processes used by LED manufacturers. These laser-based technologies offer non-contact, high-speed dicing, improving both the processing time and the chip density on a wafer [5].

Laser scribing can be performed either by nanosecond or femtosecond pulses (see Fig. 1b). In the first case, it is a thermal process based on absorption of light by the substrate. Traditionally, the industrial dicer's use nanosecond pulses either with a wavelength of $355 \mathrm{~nm}$ scribing a line, colloquially called "the kerf", across the back surface of the wafer or a wavelength of $266 \mathrm{~nm}$ for cutting from the face of it [6-9]. However, this method produces a huge amount of debris. Moreover, the use of nanosecond pulses imposes serious requirements on the cooling system due to wide areas around the kerf which are affected by the heat [10]. In addition, using UV radiation generates health concerns for the personnel handling the system. 


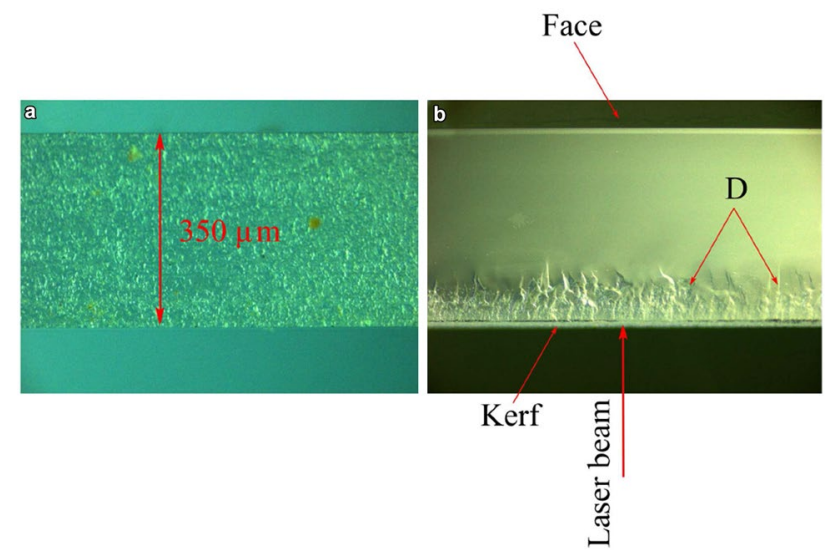

Fig. 1 The edge of the sapphire wafer through $\times 40$ objective; a sawn with the diamond saw and $\mathbf{b}$ scribed with the femtosecond pulsed laser and broken with a cleaver. Label " $D$ " indicates optical damage induced below the kerf. The roughness of the surface (a) is $\sim 0.5 \mu \mathrm{m}$, the roughness of the surface below the optical damage $(\mathbf{b})$ is $<50 \mathrm{~nm}$

Experimentally it has been found that conventional laser scribing of a sapphire surface is extremely slow and practically unreliable. Attempts to remove more than $\sim 0.1 \mu \mathrm{m}$ per scan lead either to breakage or cracking of the wafer. Indeed, reliable results have not been obtained for conventional scribing even if the scanning speed is limited to $1 \mathrm{~cm} / \mathrm{s}$ (the optimal scribing speed for our setup) and the material removed limited to $0.1 \mu \mathrm{m}$ per run. Typically, a sequence of 600 runs (few hours) produces a kerf with a depth in the range of $40-50 \mu \mathrm{m}$. After reaching this depth, the sample is frequently damaged making cleaving along the scribe unreliable. The wafer trends to form cracks and frequently the plane of the break after cleaving is not perpendicular to the surface of the wafer.

Stealth Dicing (SD) is an alternative technique, proposed by Hamamatsu Photonics K.K. A properly focused laser beam modifies the material inside the wafer creating damage inside the material. Pulse widths in both the nanosecond and picosecond range [11] have been used to dice sapphire samples. In the first case, the method is based on a periodic thermal modification of the material inside of a small volume below the surface of the wafer. The damage induced by nanosecond pulses is due to thermal ablation of the material and requires the use of laser radiation in UV range. This technique also requires a cooling system and is relatively slow due to the low repetition (typically $<50$ $\mathrm{kHz}$ ) frequency of Q-switched pulse lasers.

Sapphire absorbs a very small portion of incident light within the visible or near infrared (VR or NIR) regions. However, femtosecond pulses with an energy of tens of micro-Joules can induce the optical damage due to Coulomb explosion caused by multi-photon absorption (six photons in the case of sapphire). During this interaction, the amplitude of the applied electric field is enough to break electron bonds and destroy the crystalline structure. Hence, modification of the material can be induced using high-intensity femtosecond pulses with only nano-Joules of energy per pulse [12]. The sapphire laser pulse which is properly focused inside the sapphire converts the material of the wafer to the plasma within a few tenths of a picosecond creating local pressures $\sim 10 \mathrm{TPa}$ [13] modifying the material inside the focal spot. In this case, the part of radiation which does not surpass the damage threshold just passes through the material practically without heating it. The absence of intrinsic heating along with the low overall energy of pulse greatly reduces the damage around the irradiated area since the pulse duration is smaller than the time scale for ion-electron relaxation time [13]. In this case damage to the thin gold conductors around the kerf is mostly induced by the external parts of the focal cone and residual radiation due to metals having a lower damage threshold than the sapphire substrate and absorbing VR-NIR. In this case, the notable reduction of heat gradient around the affected zone makes the whole LED structure more stable.

In this article, we compare two processes, laser scribing, and stealth dicing. Both processes have been realized on 350 - $\mu$ m-thick sapphire wafers using an $800 \mathrm{~nm}$ fs laser with a pulse duration of $\sim 140$ fs. In contrast to previously published results [13] we have used an $\times 20$ microscopic objective with a numerical aperture (NA) of 0.55 , and a pulse energy in the range of tens of micro Joules, which is an order of magnitude higher than in [13]. Existing industrial dicer's operating in ultraviolet spectral range use objectives with high numerical aperture. These objectives induce nearly spherical damage spot with the diameter of $\sim 30 \mu \mathrm{m}$. To dice thin wafers with a thickness of less than $120 \mu \mathrm{m}$ the dicer induces one row of damage spots, meanwhile to dice a thick wafer ( $>250 \mu \mathrm{m}$ of thickness) typically two parallel rows of damage need to be made for successful sample separation. The elongated spot of optical damage induced inside the sample allows dicing of thick wafers without inducing the second row of damage spots while conserving a high edge quality. As a result, the technologies addressing these concerns, as will be discussed in this paper, are crucial for making better devices, both for research and mass production.

\section{Experimental setup}

In the experiments, we used a polished sapphire wafer with a thickness of $350 \mu \mathrm{m}$. The laser machining was performed using a Ti:Sapphire (Spectra Physics Tsunami) laser system which generates $140 \mathrm{fs}$ pulses at a $1 \mathrm{kHz}$ repetition frequency and at a center wavelength of $800 \mathrm{~nm}$. The laser has a mechanical shutter which allows delivering bursts of 
$\sim 100$ pulses in 0.1 seconds. The beam was focused using a $\times 100$, a $\times 20$ and a $\times 5$ microscope objectives with NA's of 0.55 and 0.28 , respectively. At the laser wavelength of $800 \mathrm{~nm}$, this produces a tightly focused beam with focal spot diameters of 0.74 and $3.5 \mu \mathrm{m}$, respectively, from the 3-mm collimated beam. A high-precision air bearing stage (Aerotech 5 axis, 3 linear, 2 tilt) was used to scan the sample in three dimensions according to our requirements and to align the sample (Fig. 2). The movement of the stage was controlled using a custom-built computer program. After dicing, the samples were imaged with an optical microscope and a SEM without any thinning or lapping.

The experimental setup is shown in Fig. 2. The laser power was adjusted by rotating a half-wave plate in front of

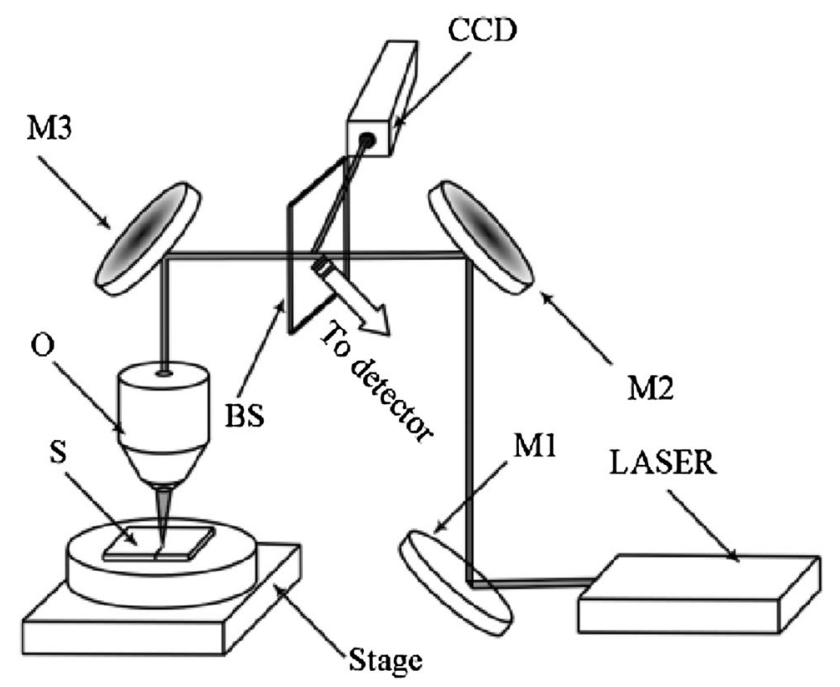

Fig. 2 Experimental setup a polarizer. A partially reflective plate was used to couple a small fraction of the laser radiation (10\%) towards a detector and also to reflect visible light back from the sample to a CCD. This imaging system was used both for alignment and for the initial inspection of the damage.

\section{Results and discussion}

\subsection{Pulse energy and depth of focus}

Sapphire has a very low absorption coefficient at $800 \mathrm{~nm}$ and hence it is possible to focus the laser energy deep inside the sapphire crystal. However, strong absorption occurs at the focus once the laser intensity exceeds the threshold for multi-photon absorption. This allows damage to be induced inside the crystal without affecting the rest of the structure. We have investigated the damage generated as a function of energy at different depths inside the sapphire.

The results of this experiment can be conveniently mapped out as shown in Fig. 3a. This figure illustrates a matrix of laser-induced modification in which the pulse energy is stepped up in the vertical direction from 1 to 30 $\mu \mathrm{J}$. In the horizontal direction, the depth of the focus was increased in $10 \mu \mathrm{m}$ steps from the top of the surface at the left towards the bottom of the surface at the right. Three different zones are clearly visible on this energy/depth map. Lower energy pulses only produce small superficial craters and do not induce damage in the body of the target. This area is labeled as "red 1" and it encloses the regimes suitable for scribing layer by layer. The area labeled as "blue 2 " shows the regimes that can be used for stealth dicing. The pulses in this regime produce superficial craters with
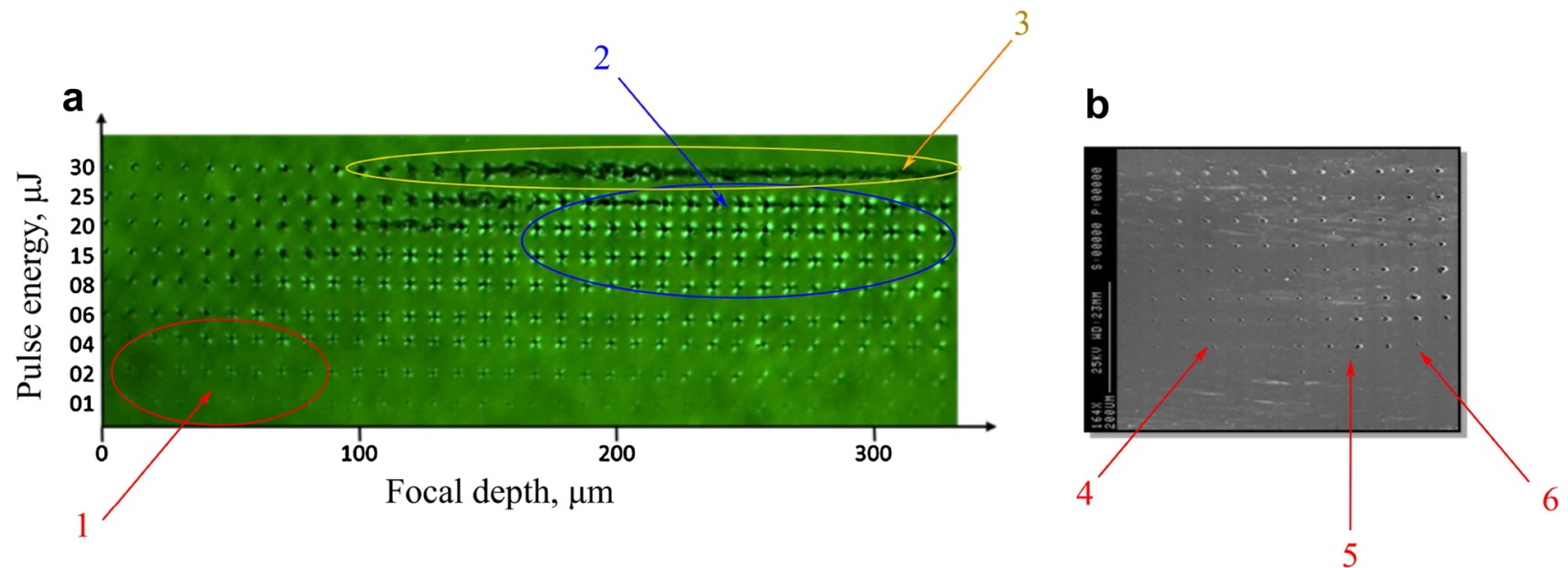

Fig. 3 A matrix of craters is showing the damage generated as a function of the laser pulse energy (vertical axis) and the depth of the focal position inside the sapphire (horizontal axis). a Optical image,

b SEM image for focal depths from 0 to $125 \mu \mathrm{m}$. In this case, the $\times$ 100 objective was used to focus the beam at each position. The exposure time was set to $100 \mathrm{~ms}$. 
a depth of less than 20 microns and also induce an internal damage (see Fig. 7). Finally, the area labeled as "yellow 3" corresponds to the excessive damage of the sample (pulse energy of $30 \mu \mathrm{J}$ ). The pulse energy needed for the regimes " 1 " and " 2 " differs by a factor of $\sim 10$ with $1-2 \mu \mathrm{J}$ aptly suited for scribing and $\sim 25 \mu \mathrm{J}$ for stealth dicing.

Figure $3 b$ shows a SEM image of the surface for the left side of the sample where the focal depth is varied from $0 \mu$ $\mathrm{m}$ to $125 \mu \mathrm{m}$ which can be compared with the microscope image in Fig. 3a. For low energies, the pulses only induced superficial damage (label "4" in Fig. 3b, and Fig. 4a). If the energy of the pulse is high enough (label " 5 " in Fig. 3b) then the pulse "drilled" a small hole (compare with Fig. 4b) with a depth up to $10 \mu \mathrm{m}$; as the focal depth was increased, this superficial damage, as well as the hole, disappeared (label "6" in Fig. 3b).

The damage induced using a pulse energy in the range of $1-2 \mu \mathrm{J}$ is illustrated in Fig. 4a. In this case, the crater has an oval shape and a depth of $\sim 2 \mu \mathrm{m}$ and appears quite suitable for conventional scribing. It is clearly visible that this crater is formed principally because of chipping of the sapphire surface, during a process which is probably similar to an explosion. The shape of the crater implies that the pulse energy was very close to the damage threshold. The crater for the regime using a pulse energy of $6 \mu \mathrm{J}$ is neat with a circular surface geometry of diameter $\sim 2 \mu \mathrm{m}$ and depth which can reach $\sim 5 \mu \mathrm{m}$, depending on the focal position. In this case, the superficial chipping is minor and the crater is a clean hole. This probably means that the pulses have enough energy to evaporate a considerable amount of material. The plasma was evacuated from the epicenter

practically without creating a shock wave. Finally, Fig. 4c shows the damage caused using a pulse energy of $25 \mu \mathrm{J}$. Here the damage was much deeper $\sim 15 \mu \mathrm{m}$ leaving a crater with the dimensions of $\sim 25 \mu \mathrm{m}$. In this case, considering the excitation conditions and from the appearance of the crater we believe that it is likely that all of the superficial damage was due to coulomb explosion and, moreover, the surface chipping was minimal. The shape of the crater matches the symmetry of the sapphire crystalline structure the surface of the wafer parallel to c-plane (0001), forming an equilateral triangle with angles of $\sim 60^{\circ}$.

In conclusion, for low-energy pulses, the visible damage will normally be confined to a small region around the focal position (Fig. 4b). However, as the energy is increased, the greater damage will generate stress which radiates away from the focal region. If the focal position is sufficiently close to a surface, the induced damage can leave a mark. The maximum depth at which damage occurs increases with pulse energy as it is illustrated in Fig. 4c. At the highest pulse energy $(>6 \mu \mathrm{J})$, the damage occurs over the whole range of depths (see Fig. 3).

\subsection{Scribing}

During the scribing process, the damage on the sapphire surface is induced by pulses creating a continuous kerf due to overlapping craters. In this way, after one run the laser beam traces a trench on the surface removing a few micrometers of the material. Repeating this procedure as many times as it is necessary it is possible to "saw" or scribe the wafer in the same fashion as the diamond tip
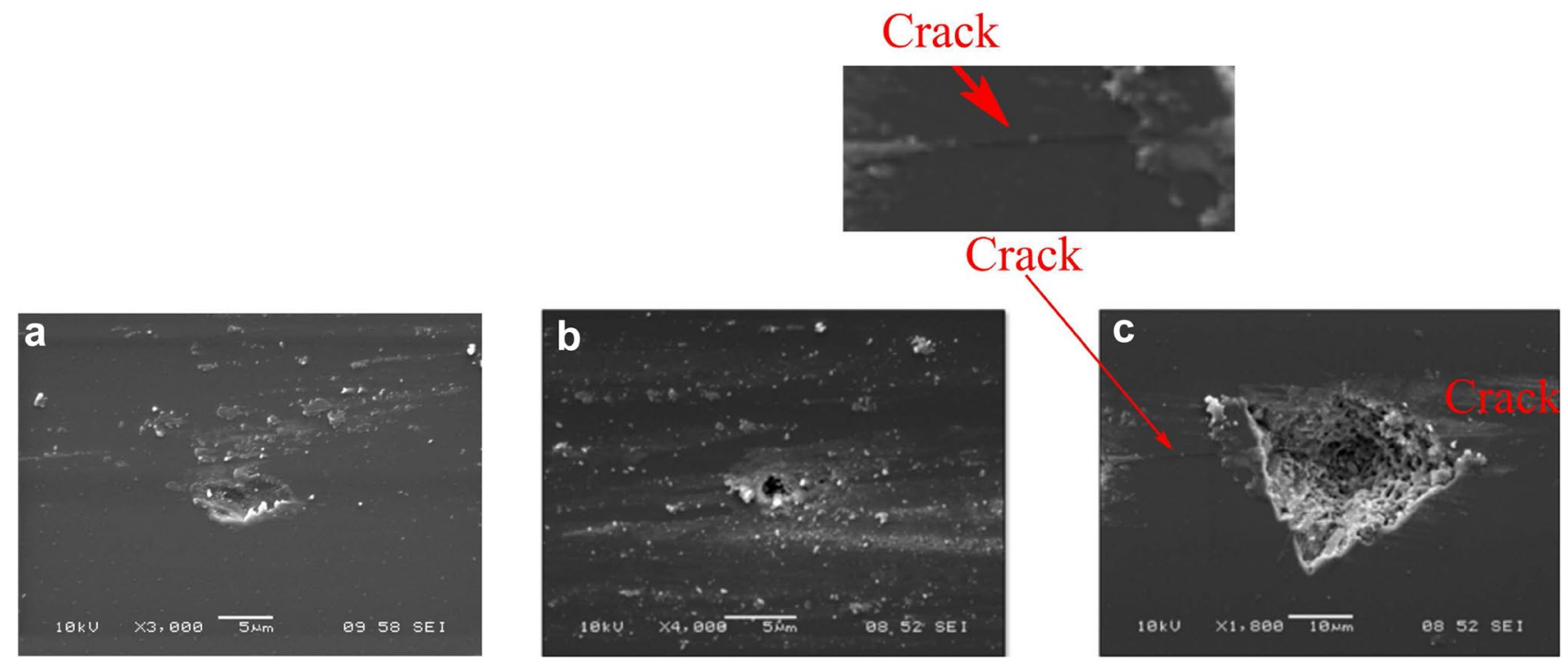

Fig. 4 SEM images of regimes of interest; $\mathbf{a}$ energy of $2 \mu \mathrm{J}$ and the focal depth of $0 \mu \mathrm{m}$ (depth of the crater $<5 \mu \mathrm{m}$ ); $\mathbf{b}$ energy of $6 \mu \mathrm{J}$ and the depth of the focus of $25 \mu \mathrm{m}$ (depth of the crater $\sim 5 \mu \mathrm{m}$ ); $\mathbf{c}$ energy of $25 \mu \mathrm{J}$ and the depth of the focus of $200 \mu \mathrm{m}$ (depth of the crater $15 \mu \mathrm{m})$. The insert shows a close-up of the area illustrating the crack induced in the wafer 
does. When the kerf is deep enough the wafer can be broken using a cleaver in the same way as a glass plate.

To evaluate thermal damage during the scribing we sprayed oil over the surface of a wafer before laser scribing. The results of this experiment are shown in Fig. 5. The beam generated a feature with a width of $\sim 45 \mu \mathrm{m}$ in the surface of the sample (the black line in Fig. 5) and evaporated the oil film around the mark over a distance of approximately $200 \mu \mathrm{m}$. The kerf was surrounded by a wall of debris with a width of $\sim 50 \mu \mathrm{m}$.

A close up of the black line is illustrated in Fig. 6a. In this experiment, the wafer had a structure printed on it. The structure consisted of gold strips with a width of $\sim 200 \mu \mathrm{m}$ printed on the bottom side of the wafer (Fig. 6). This structure was emulating the gold contacts of a LED. The beam generated a kerf with a width of $\sim 4 \mu \mathrm{m}$ in the surface of the wafer but also damaged the gold contacts, practically

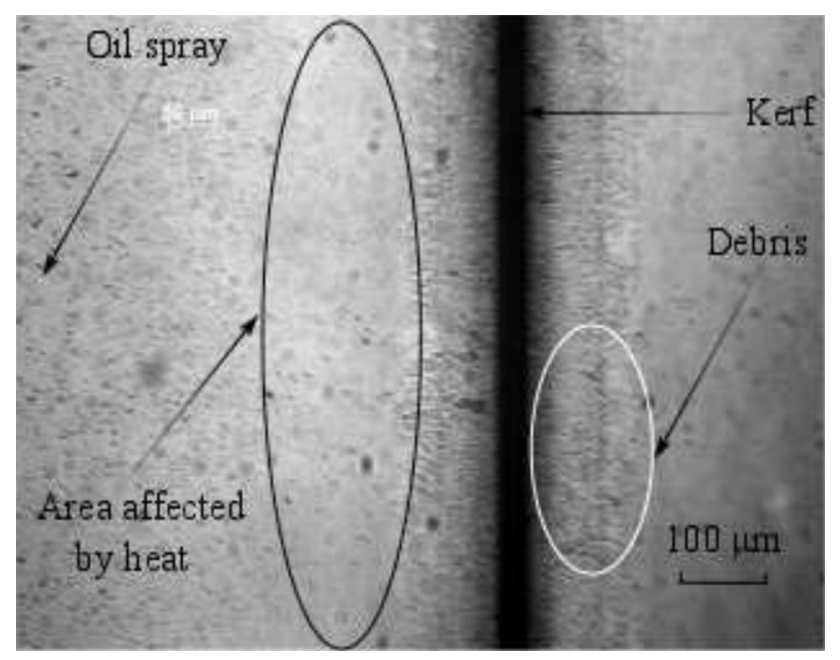

Fig. 5 The thermal damage induced by the laser beam after 600 runs over the surface of the sample destroying them. The width of the damage was measured to be $\sim 45 \mu \mathrm{m}$.

During this set of experiments it was found that the kerf could be produced evenly across the whole wafer if required, maintaining its width at $\sim 4-5 \mu \mathrm{m}$. However, this procedure was relatively unstable because the wafer frequently became bent along the kerf, probably due to the heavy damage induced in the sapphire structure by the laser beam. After scribing a few $\mu \mathrm{m}$ depth, the sample was cleaved. The face of the wafer after cleaving is shown in Fig. 6b. In the face of the wafer, we can observe a series of cracks. The structure of these cracks reveals a series of stress points lying at a depth of $\sim 200 \mu \mathrm{m}$ (from the beam side) and separated by $50-70 \mu \mathrm{m}$ from each other. Also, it was found that the depth where these stress points appear, as well as their separation, depends on the kerf depth. When the kerf is made deeper, the stress points become less visible and completely disappear when the depth of the kerf surpasses $\sim 10 \%$ the width of the wafer (see Fig. 1b). It also depends on the width of the sample. All our attempts to scribe a real LED structure with a street width of $40 \mu \mathrm{m}$ were futile because all the LED structures at the row next to the kerf were damaged irreversibly.

\subsection{Stealth dicing}

As it was expected, when the position of damage areas inside of the wafer match with the positions of the stress points, observed in the first series of experiments, and the energy of the pulses was high enough, the wafer was split along the line of craters (one of these craters is shown in Fig. 4c).

The quality of the surface after dicing was checked using an atomic force microscopy (AFM) and typical results are shown in Fig. 7. It was observed that the cleaved surface below the damaged area (the beam was directed downward), i.e. towards the bottom surface, has a very low
Fig. 6 a The damage induced in the gold coating after 600 rounds of scribing and $\mathbf{b}$ the face of the wafer after cleaving

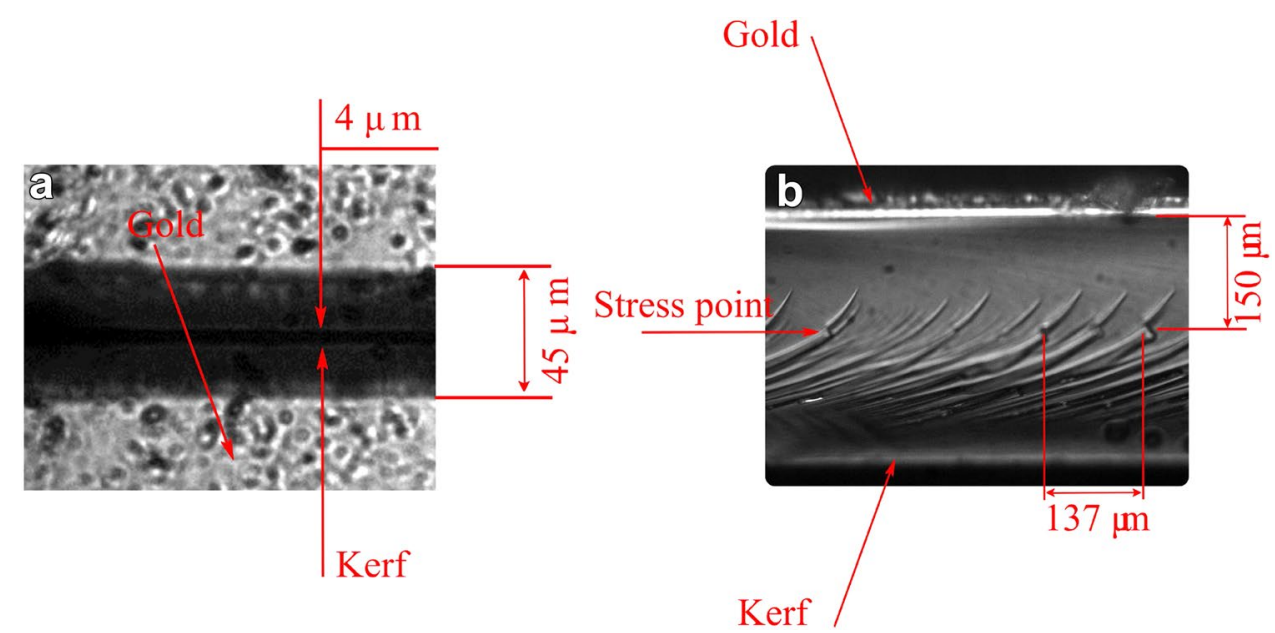




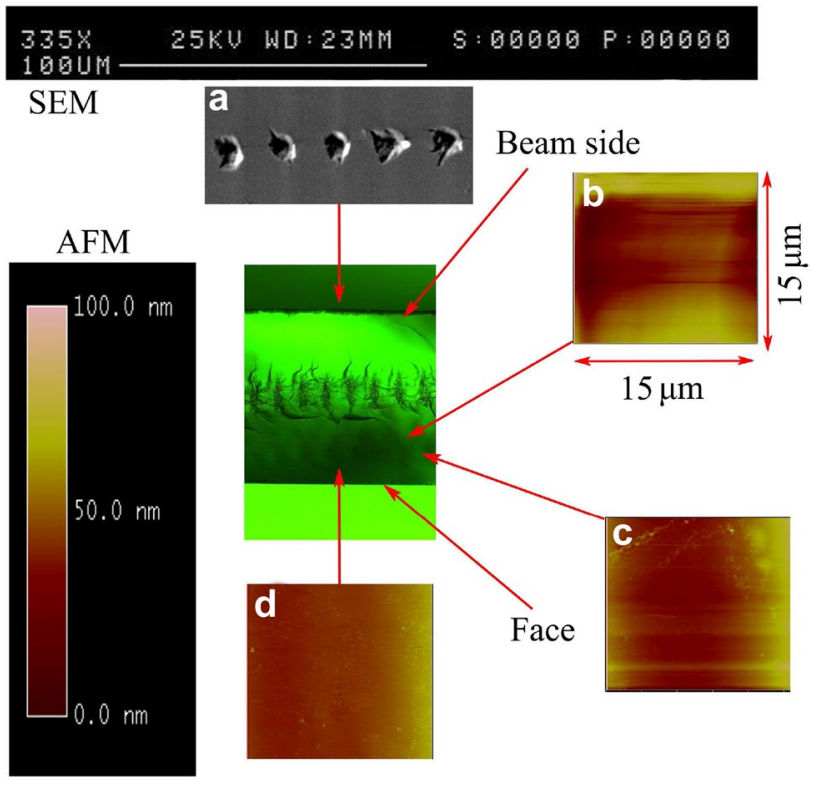

Fig. 7 Stealth dicing using $25 \mu \mathrm{J}$ pulses and an $\times 100$ objective. The insets illustrate the AFM and SEM results. Chipping is visible on the beam side. The distance between the shots was $50 \mu \mathrm{m}$. One hundred pulses per shot were delivered

roughness in the range of 20-50 nm (Fig. 7b-d), which corresponds to a thickness of around 100 atomic layers. The surface far from the damaged area has ripples with a width of $10-30 \mu \mathrm{m}$ and a height of $\sim 30 \mathrm{~nm}$. The height of these ripples is below the diffraction limit for blue/violet light and hence they should not affect the beam quality. Also, a slight superficial damage of the edges is observed on the beam side (Fig. 7a) but is limited only to the surface. In any case, this damage was considerably smaller than in the case of mechanical scribing.

For an array of GaN LEDs fabricated on a sapphire substrate, minimizing the amount of damage induced by the gold layer is a very crucial issue. Hence, the distance between pulses was set to be $50 \mu \mathrm{m}$ which can be compared with the typical width of the LED devices which is around of $200 \mu \mathrm{m}$ (3-5 shots per device edge). Using these conditions, the damage to the gold contacts was minimized.

Figure 8 shows optical images of the surface following stealth dicing performed at two different pulse energies. In Fig. 8a, the pulse energy was $25 \mu \mathrm{J}$ and a $\times 100$ microscope objective lens was used to focus the laser at a depth of $330-270 \mu \mathrm{m}$ from the top surface. For these energy pulses, the sample was exposed to the laser in $50 \mu \mathrm{m}$ steps for a period of $100 \mathrm{~ms}$. The minimum exposure time was limited by the speed of the shutter blocking the beam. At this exposure time, the laser was delivering $\sim 100$ pulses during the time that the shutter was open. In this case, it was found that the surface opposite the beam was heavily damaged by the pulse if the focal depth was greater than $270 \mu$ $\mathrm{m}$ but otherwise the surface appeared clean. This experiment shows that it is probably better to select a microscope objectives with a small numerical aperture $(0.55$ in our case) which induces only one row of defects at the depth of $\sim 60 \%$ of the sample thickness rather than use an objective with a high numerical aperture and induce two rows of defects at depths of $\sim 30$ and $80 \%$ of the sample thickness. In contrast to $\mathrm{Si}$, we found that for sapphire samples pulses focused at a depth of $80 \%$ of the wafer thickness damaged the surface.

The second experiment (Fig. 8b) was made to investigate contrasting cleaving conditions. In this case, the damage mechanism was different and the shock wave was not generated (or at least it was notably smaller than in the previous case). Pulses with the energy of $8 \mu \mathrm{J}$ (Fig. 8b) still have sufficient energy to damage sapphire by enhanced electronic excitation; however, the damaged area was too small to bridge the $50 \mu \mathrm{m}$ gap between the exposed regions and allow dicing using the same method as was used for high-energy pulses. The sample was scanned 500 times, to generate the result shown in Fig. 8b. The sample did not split by itself and we used the cleaver to break it. Although it was quite easy to break the sample, the surface opposite
Fig. 8 a The stealth dicing realized with $25 \mu \mathrm{J}$ pulses, $X$ 100 objective, and variable focal depth; $\mathbf{b}$ the stealth dicing realized with $8 \mu \mathrm{J}$ pulses, $x$ 5 objective and focal depth of $170 \mu \mathrm{m}$. In the last case, it was necessary to perform 500 runs and then break the wafer using the cleaver

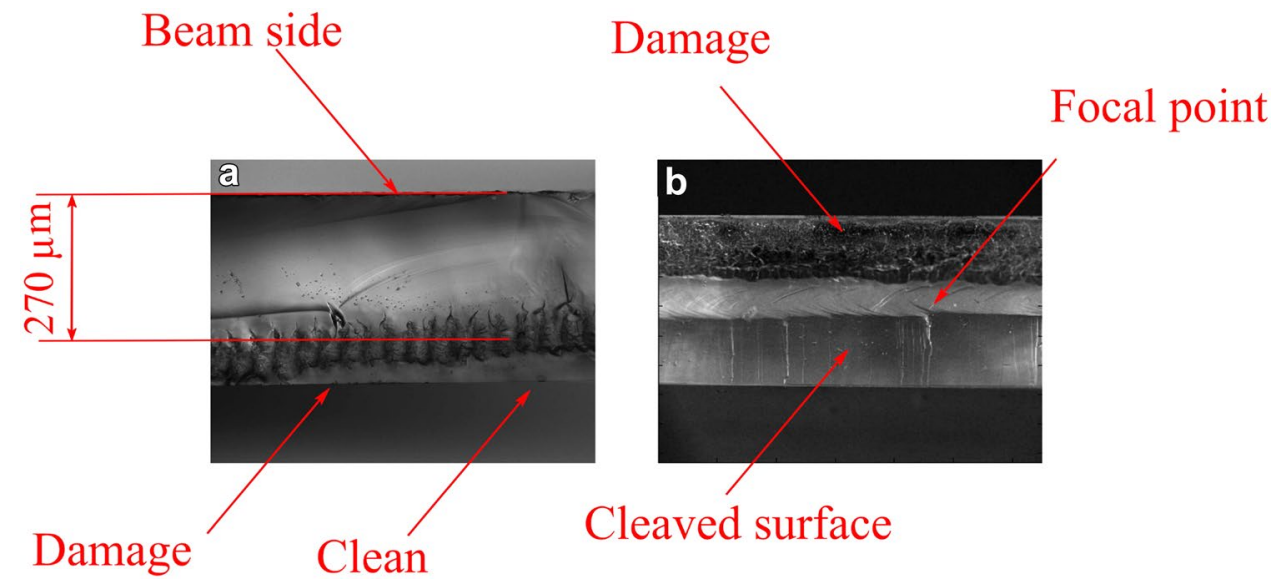


to the beam showed an unclean stair-like structure. The comparison between these two experiments confirms our hypothesis that the shock wave generated in the sample during Coulomb explosion is a necessary component for clean separation of the sapphire wafer.

\section{Conclusion}

In conclusion, the scribing technique was not found to be useful for dicing LED structures. This was because the process was slow and because it induced damage in the LEDs. During the dicing of real GaN devices, the damage to the gold conductors was limited to a distance of $\sim 20 \mu \mathrm{m}$ around the edges; however, the radius of the damage induced by the device was estimated to be up to $\sim 300 \mu \mathrm{m}$. The portion of the "dead" devices in the rows adjacent to the kerf after dicing was $>80 \%$. However, this technique can still be useful to get the information about the stress centers in the body of a wafer (the position and the separation of these centers depend on the wafer thickness).

We conclude that successful "stealth dicing" of sapphire wafers is possible using NIR wavelengths; moreover, generating an elongated damage spot (using a microscope objective with a low NA) allows the dicing of thick sapphire samples $(350 \mu \mathrm{m})$ inducing only one row of defects. Altering the damage profile, by changing the numerical aperture of the focusing lens should allow the conditions for stealth dicing to be optimized for samples with different thickness. The AFM analysis has demonstrated that the quality of the edge opposite to the side of the beam entrance was good enough for manufacturing of blue/violet LEDs. In addition, as only one scan line was required at the higher pulse energy, using a high repetition rate mode-locked laser this technique has the scope to be fast, for example, using a fs laser with $1 \mathrm{MHz}$ repetition rate, dicing speed of $\sim 50 \mathrm{~m} / \mathrm{s}$ could possibly be achieved for craters separated by a distance of $50 \mu \mathrm{m}$.

Acknowledgements This work was supported by the West Midlands European Regional Development Fund (ERDF) project, Leverhulme Trust (Grant ref: RPG-2014-304), FP7-PEOPLE-2012-IAPP (Project GRIFFON, No. 324391) and FP7-NEWLED project (Grant No. 318388).

Open Access This article is distributed under the terms of the Creative Commons Attribution 4.0 International License (http:// creativecommons.org/licenses/by/4.0/), which permits unrestricted use, distribution, and reproduction in any medium, provided you give appropriate credit to the original author(s) and the source, provide a link to the Creative Commons license, and indicate if changes were made.

\section{References}

1. G.D. Boyd, H. Kogelnik, Bell Syst. Techn. J. 41(4), 1347 (1962). doi:10.1002/j.1538-7305.1962.tb03281.x. http://ieeexplore.ieee. org/lpdocs/epic03/wrapper.htm?arnumber=6773469

2. K. Itaya, M. Onomura, J. Nishio, L. Sugiura, S. Saito, M. Suzuki, J. Rennie, S.y. Nunoue, M. Yamamoto, H. Fujimoto, Y. Kokubun, Y. Ohba, G.i. Hatakoshi, M. Ishikawa, Jpn. J. Appl. Phys. 35(Part 2, No. 10B), L1315 (1996). doi:10.1143/JJAP.35. L1315. http://stacks.iop.org/1347-4065/35/L1315

3. V. Pishchik, L.A. Lytvynov, E.R. Dobrovinskaya, in Sapphire: Material, manufacturing, Applications (Springer US, Boston, MA, 2009), chap. 2, pp. 55-176. doi:10.1007/978-0-387-85695-7

4. K. Matsumaru, A. Takata, K. Ishizaki, Sci. Technol. Adv. Mater. 6(2), 120 (2005). doi:10.1016/j.stam.2004.11.002

5. M. Mendes, J. Fu, C. Porneala, X. Song, M. Hannon, J. Sercel, in Proc. SPIE 7584, Laser Applications in Microelectronic and Optoelectronic Manufacturing XV, ed. by $\mathrm{H}$. Niino, M. Meunier, B. Gu, G. Hennig (2010), p. 75840T. doi:10.1117/12.843815. http://proceedings.spiedigitallibrary.org/proceeding.aspx? doi $=10.1117 / 12.843815$

6. G.Y. Mak, E.Y. Lam, H.W. Choi, J. Vacuum Sci. Technol. B: Microelectron. Nanometer Struct. 28(2), 380 (2010). doi:10.1116/1.3359593. http://scitation.aip.org/content/avs/ journal/jvstb/28/2/10.1116/1.3359593

7. H.M. Lam, M. Hong, S. Yuan, T.C. Chong, in Proc. SPIE 4830, Third International Symposium on Laser Precision Microfabrication, ed. by I. Miyamoto, K.F. Kobayashi, K. Sugioka, R. Poprawe, H. Helvajian (2003), p. 114. doi:10.1117/12.486561. http://proceedings.spiedigitallibrary.org/proceeding.aspx? doi=10.1117/12.486561

8. E. Illy, M. Knowles, E. Gu, M. Dawson, Appl. Surf. Sci. 249(14), 354 (2005). doi:10.1016/j.apsusc.2004.12.033. http://linkinghub.elsevier.com/retrieve/pii/S0169433204018306

9. A. Tamhankar, R. Patel, J. Laser Appl. 23(3), 032001 (2011). doi:10.2351/1.3589243. http://scitation.aip.org/content/lia/ journal/jla/23/3/10.2351/1.3589243

10. T. Nilsson, F. Wagner, R. Housh, B. Richerzhagen, in Proc. SPIE 5366, Light-Emitting Diodes: Research, Manufacturing, and Applications VIII, ed. by S.A. Stockman, H.W. Yao, E.F. Schubert (2004), p. 200. doi:10.1117/12.529012. http://proceedings. spiedigitallibrary.org/proceeding.aspx?doi=10.1117/12.529012

11. Z. Yiyun, G. Enqing, L. Zhi, W. Tongbo, L. Jing, Y. Xiaoyan, W. Guohong, IEEE Photon. Technol. Lett. 24(4), 243 (2012). doi:10.1109/LPT.2011.2177251. https://www.osapublishing.org/ oe/abstract.cfm?uri=oe-20-6-6808

12. S. Juodkazis, H. Misawa, Appl. Phys. A 93(4), 857 (2008). doi:10.1007/s00339-008-4763-0

13. S. Juodkazis, K. Nishimura, S. Tanaka, H. Misawa, E.G. Gamaly, B. Luther-Davies, L. Hallo, P. Nicolai, V.T. Tikhonchuk, Phys. Rev. Lett. 96(16), 166101 (2006). doi:10.1103/ PhysRevLett.96.166101 